\title{
Simulation of Dislocation Annihilation by Cross-Slip
}

\author{
P. PAUS $\check{S}^{a, *}$, M. Beneš ${ }^{a}$ AND J. KRATOChVÍL ${ }^{b}$ \\ ${ }^{a}$ Department of Mathematics, Faculty of Nuclear Sciences and Physical Engineering \\ Czech Technical University in Prague, Trojanova 13, Prague, Czech Republic \\ ${ }^{b}$ Department of Physics, Faculty of Civil Engineering, Czech Technical University in Prague \\ Thákurova 7, Prague, Czech Republic
}

\begin{abstract}
This contribution deals with the numerical simulation of dislocation dynamics, their interaction, merging and changes in the dislocation topology. The glide dislocations are represented by parametrically described curves moving in slip planes. The simulation model is based on the numerical solution of the dislocation motion law belonging to the class of curvature driven curve dynamics. We focus on the simulation of the cross-slip of two dislocation curves where each curve evolves in a different slip plane. The dislocations evolve, under their mutual interaction and under some external force, towards each other and at a certain time their evolution continues outside slip planes. During this evolution the dislocations merge by the cross-slip occurs. As a result, there will be two dislocations evolving in three planes, two planes, and one plane where cross-slip occurred. The goal of our work is to simulate the motion of the dislocations and to determine the conditions under which the cross-slip occurs. The simulation of the dislocation evolution and merging is performed by improved parametric approach and numerical stability is enhanced by the tangential redistribution of the discretization points.
\end{abstract}

PACS: 02.70.Bf, 61.72.Lk, 02.30.Jr

\section{The model}

Dislocation cross-slip is one of key processes of crystal plasticity. The most important cross-slip models are reviewed in [1]. The present simulation is focused on the annihilation of screw dislocation parts by cross-slip as an important factor in the generation and dynamics of persistent slip bands. In the bands screw parts of glide dislocations moving in channels of low dislocation density mutually annihilate when the distance between their respective slip planes falls below a critical limit. The remaining edge parts are stored in multipolar walls. The annihilation by cross-slip is governed by the line tension, the applied stress in the channels and the interaction force between dislocations. In the present simulation a dissociation of the glide dislocations both in their slip planes and the cross-slip plane is neglected. In discrete dislocation dynamics modeling dislocations as "zigzag" lines jumping on a discrete network cross-slip is a standard ingredient [2]. This article describes the incorporation of the annihilation by cross-slip into the discrete dislocation dynamics by considering dislocations as moving smooth curves. This approach is a mathematically challenging alternative.

The interaction of dislocations can be approximately described using the curvature flow. We consider perfect dislocation curves with the Burgers vector $\boldsymbol{b}=(0,0, b)$

* corresponding author; e-mail: petr.paus@fjfi.cvut.cz oriented in the $x$-direction of the $x, y, z$ coordinate system. The dislocation curve motion $\Gamma$ is located in a slip plane, identified as $x z$-plane. The glide of dislocation is governed by the relaxation law in the form of the mean curvature flow equation in the direction of the normal vector to the dislocation

$$
B v=L \kappa+b\left(\tau_{\text {app }}+\tau_{\text {int }}\right),
$$

where $B$ is a drag coefficient, and $v(\boldsymbol{x}, t)$ is the normal velocity of a dislocation at $\boldsymbol{x} \in \Gamma$ and time $t$. The term $L \kappa$ represents self-force expressed in the line tension approximation as the product of the line tension $L$ and local curvature $\kappa(x, t)$. The term $\tau_{\text {app }}$ represents the local shear stress acting on the dislocation segment produced by the bulk elastic field. The term $\tau_{\text {int }}$ represents interaction force between dislocations. In our simulations, we consider the "stress controlled regime" where the applied stress in the channel is kept uniform. In the slip plane, the applied stress $\tau_{\text {app }}$ is the same at each point of the line and for numerical computations we use $\tau_{\text {app }}=$ const. The "strain controlled regime" analyzed in [3] could be an alternative.

\section{Parametric description}

The motion law (1.1) in the case of dislocation dynamics is treated by parameterization where the planar curve $\Gamma(t)$ is described by a smooth time-dependent vector function $\boldsymbol{X}: S \times I \rightarrow \mathbb{R}^{2}$, where $S=[0,1]$ is a fixed interval for the curve parameter and $I=[0, T]$ is the 
time interval. The curve $\Gamma(t)$ is then given as the set $\Gamma(t)=\left\{\boldsymbol{X}(u, t)=\left(X^{1}(u, t), X^{2}(u, t)\right), u \in S\right\}$.

The evolution law (1.1) is transformed into the parametric form as follows. The unit tangential vector $\boldsymbol{T}$ is defined as $\boldsymbol{T}=\partial_{u} \boldsymbol{X} /\left|\partial_{u} \boldsymbol{X}\right|$. The unit normal vector $\boldsymbol{N}$ is perpendicular to the tangential vector and $\boldsymbol{N} \cdot \boldsymbol{T}=0$ holds. The curvature $\kappa$ is defined as

$$
\kappa=\frac{\partial_{u} \boldsymbol{X}^{\perp}}{\left|\partial_{u} \boldsymbol{X}\right|} \cdot \frac{\partial_{u u} \boldsymbol{X}}{\left|\partial_{u} \boldsymbol{X}\right|^{2}}=\boldsymbol{N} \cdot \frac{\partial_{u u} \boldsymbol{X}}{\left|\partial_{u} \boldsymbol{X}\right|^{2}},
$$

where $\boldsymbol{X}^{\perp}$ is a vector perpendicular to $\boldsymbol{X}$. The normal velocity $v$ is defined as the time derivative of $\boldsymbol{X}$ projected into the normal direction, $v=\partial_{t} \boldsymbol{X} \partial_{u} \boldsymbol{X}^{\perp} /\left|\partial_{u} \boldsymbol{X}\right|$. Equation (1.1) can be written as

$$
B \partial_{t} \boldsymbol{X}=L \frac{\partial_{u u} \boldsymbol{X}}{\left|\partial_{u} \boldsymbol{X}\right|^{2}}+b\left(\tau_{\mathrm{app}}+\tau_{\mathrm{int}}\right) \frac{\partial_{u} \boldsymbol{X}^{\perp}}{\left|\partial_{u} \boldsymbol{X}\right|} .
$$

This equation is accompanied by the periodic boundary conditions for closed curves, or by the fixed-end boundary condition for open curves, and by the initial condition. These conditions are considered similarly as in [4]. For long time computations with time and space variable force, the algorithm for curvature adjusted tangential velocity is used. Details are described in [5]. To incorporate a tangential redistribution, a tangential term $\alpha$ has to be added to Eq. (2.1).

$$
\begin{gathered}
B \partial_{t} \boldsymbol{X}=L \frac{\partial_{u u} \boldsymbol{X}}{\left|\partial_{u} \boldsymbol{X}\right|^{2}}+L \alpha \frac{\partial_{u} \boldsymbol{X}}{\left|\partial_{u} \boldsymbol{X}\right|} \\
+b\left(\tau_{\text {app }}+\tau_{\text {int }}\right) \frac{\partial_{u} \boldsymbol{X}^{\perp}}{\left|\partial_{u} \boldsymbol{X}\right|} .
\end{gathered}
$$

For numerical approximation we consider a regularized form of (2.2). The equation is then solved by means of matrix factorization. Since there are two components of $X$, two linear systems are solved in each time step.

In case of the single dislocation dynamics, the mathematical model of curve evolution is $2 \mathrm{D}$ only. However, the cross-slip phenomenon requires a $3 \mathrm{D}$ configuration to be considered (two slip planes and one cross-slip plane). Our idea is to perform a linear mapping from a virtual plane to $3 \mathrm{D}$ according to Fig. 1 . The curve motion is computed in the virtual plane and then mapped to the real physical configuration of the clip and cross-slip planes. The angle $\alpha$ is $\pi / 4$ according to the features of the crystalline lattice.

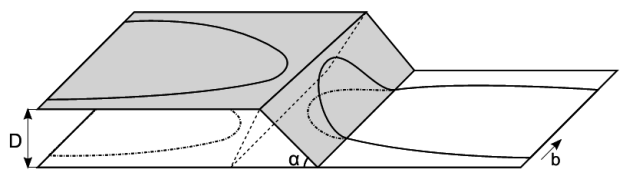

Fig. 1. Geometry of the model.

Since the parametric approach cannot handle topological changes, a modified algorithm is used (see $[6,7]$ ).

The numerical simulations were performed for copper under the following set of parameters (Table).
Parameters of numerical simulations.

\begin{tabular}{l|l}
\hline \hline Burgers vector magnitude & $b=0.25 \mathrm{~nm}$ \\
line tension & $L=2 \mathrm{nN}$ \\
drag coefficient & $B=1.0 \times 10^{-5} \mathrm{~Pa} \mathrm{~s}$ \\
applied stress & $\tau_{\text {app }}=20 \mathrm{MPa}$ \\
plain distance & $D=50 \mathrm{~nm}$ \\
cross-slip plane angle & $\alpha=\pi / 4$
\end{tabular}

The example in Fig. 2 shows the simulation of the annihilation by cross-slip. Initially the dislocations move towards each other under the external stress $\tau_{\text {app }}=$ $20 \mathrm{MPa}$. When they approach each other the interaction force rises and they are pulled together in the cross-slip plane. Finally they annihilate by cross-slip and stick to the channel walls. In reality this mechanism is hindered by dissociation of dislocation core but the presented approximation does not consider such phenomenon yet.

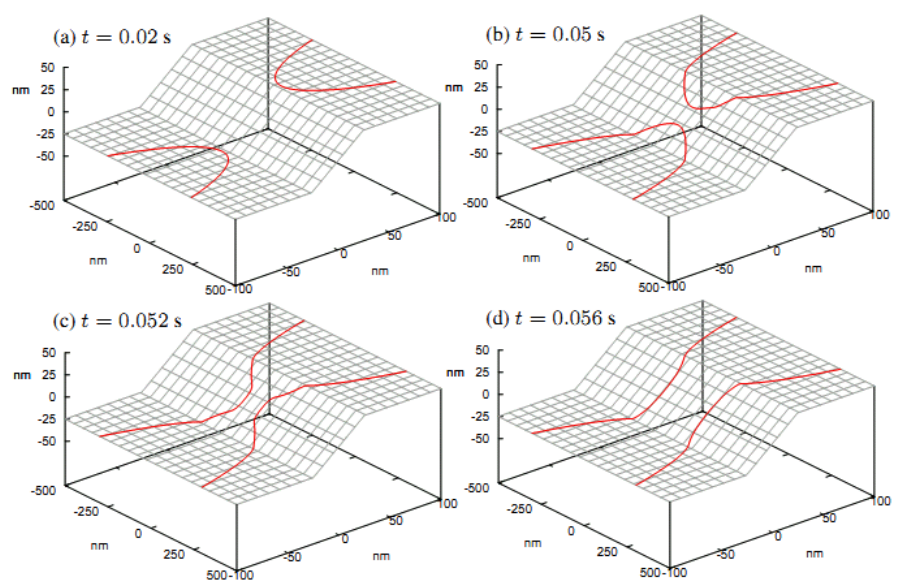

Fig. 2. Annihilation by cross-slip, $\tau_{\text {app }}=20 \mathrm{MPa}$, $t \in(0,0.06)$, dislocation curve discretized by $M=300$ nodes.

\section{Conclusion}

This work presents the numerical simulation of dislocation annihilation by cross-slip. Two dislocations move in a channel under external stress field and by atractive forces between them. When the interaction force is high enough, the cross-slip occurs followed by the annihilation. The model is very fast and can be easily modified for more dislocations in the same channel. Our next goal is to determine critical distance of cross-slip planes for which the cross-slip occurs. Also the physical model will be further developed and improved.

\section{Acknowledgments}

The research has been supported by the grants VZ-MŠMT 6840770021, GAČR - P201/10/0357, SGS 10-811610 , MSM No. 6840770100 "Applied Mathematics in 
Technical and Physical Sciences" and by the project No. LC06052 "Nečas Center for Mathematical Modelling" of the Ministry of Education, Youth and Sport of the Czech Republic.

\section{References}

[1] W. Püschl, Prog. Mater. Sci. 47, 415 (2002).

[2] L. Kubin, B. Devincre, T. Hoc, Philos. Mag. 86, 4023 (2006).

[3] J. Křišţan, J. Kratochvíl, V. Minárik, M. Beneš, in: Frontiers in numerical analysis. 10th LMS-EPSRC numerical analysis summer school, Durham (UK), July 7-19, Eds. J.F. Blowey, A.W. Craig, T. Shardlow, Springer, Berlin 2002, p. 63.
[4] K. Deckelnick, G. Dziuk, Frontiers Num. Anal. 63, (2002).

[5] D. Ševčovič, S. Yazaki, http://www.iam.fmph.uniba.sk/institute/ sevcovic/papers/cl39.pdf, arXiv:0711.2568 (2007).

[6] P. Pauš, M. Beneš, Kybernetika 45, 591 (2009).

[7] V. Minárik, M. Beneš, J. Kratochvíl, J. Appl. Phys. 107, 061802 (2010). 